HUTP-06/A0036

\title{
On the existence and dynamics of braneworld black holes
}

\author{
A. Liam Fitzpatrick, Lisa Randall, Toby Wiseman \\ Jefferson Physical Laboratory, Harvard University \\ Cambridge MA 02138, USA \\ E-mail: fitzpatr@fas.harvard.edu, randall@physics.harvard.edu, \\ twiseman@fas.harvard.edu
}

\begin{abstract}
:
Based on holographic arguments Tanaka and Emparan et al have claimed that large localized static black holes do not exist in the one-brane Randall-Sundrum model. If such black holes are time-dependent as they propose, there are potentially significant phenomenological and theoretical consequences. We revisit the issue, arguing that their reasoning does not take into account the strongly coupled nature of the holographic theory. We claim that static black holes with smooth metrics should indeed exist in these theories, and give a simple example. However, although the existence of such solutions is relevant to exact and numerical solution searches, such static solutions might be dynamically unstable, again leading to time dependence with phenomenological consequences. We explore a plausible instability, suggested by Tanaka, analogous to that of Gregory and Laflamme, but argue that there is no reliable reason at this point to assume it must exist.
\end{abstract}




\section{Contents}

1. Introduction and summary 1

2. Existence of localized static black holes 3

2.1 A counter-example to the claim of no static black holes

2.2 Reduction of low energy degrees of freedom from strong coupling

2.3 Geometry of localized black holes 9

3. Stability of localized static black holes 11

3.1 Gregory-Laflamme instability 12

3.2 Holographic interpretation of instabilities

3.3 A possible instability

3.4 Entropy Balance 14

1. Effective Theory Interpretation 16

\section{Introduction and summary}

In the Randall-Sundrum one-brane model (RS2), a five-dimensional warped spacetime with a single Minkowski brane and brane-localized matter, linear perturbations of the Minkowski brane and AdS bulk appear to a brane observer to be those of a four-dimensional gravity theory up to energies set by the AdS curvature scale. Hence this model provides a low-energy dimensional reduction for brane observers, even though the extra dimension is not compact. However, it is not clear that the RS2 model exactly reproduces four-dimensional gravity at the nonlinear level. In this regard, the study of black holes is very interesting. Tanaka and Emparan et al. [1, 2, 3] have argued using holography that large black holes localized on the brane might behave very differently from four-dimensional ones, decaying classically much faster than the conventional quantum Hawking evaporation rate. This would allow this five-dimensional theory to be distinguished from four-dimensional gravity at low energies, even though linear perturbations about a flat brane do not distinguish them until energies comparable to the AdS scale.

This possibility might seem puzzling from the perspective of a four-dimensional effective theory, which contains a four-dimensional graviton mode with the correct four-dimensional gravitational interactions. However, since the extra dimension is not compact, the spectrum of Kaluza-Klein (KK) modes is continuous down to zero energy. While for linear fluctuations of the flat brane and bulk, one may still construct a four-dimensional effective theory, the validity 
of this theory beyond the linear level is unclear. In particular we will see that for a non-linear background, such as a black hole, the mass spectrum of the KK modes becomes significantly altered for modes with mass around the black hole temperature, thereby distinguishing the effective theory from pure four-dimensional gravity. The nature of these strong effects will be critical to determining if a static stable solution exists.

Tanaka and Emparan et al. [1, 2] provided evidence that classical bulk geometries correspond in the holographic dual to quantum-corrected black holes. This dual theory is 4-d gravity coupled to a gauge theory and matter fields with $N$ colors, taken in the large $N$ 't Hooft limit, with large 't Hooft coupling [6, 7, 8, 9, 10, 11. They noted that an interesting question arises in the 4-d holographic theory when one considers Hawking radiation due to the black hole into gauge theory fields. If radiation from a free field leads to an evaporation rate $\sim \hbar$, then radiation from the $O\left(N^{2}\right)$ fields of the holographic dual theory may yield an effect going as $\sim N^{2} \hbar$, which would then persist in the large $N$ limit even as $\hbar \rightarrow 0$. Tanaka and Emparan et al. pointed out such spontaneous quantum radiation in the 4-d holographic theory would correspond to a classical 5-d process in the bulk, which would imply a 5-d black hole localized to the brane should always classically radiate, and hence cannot be static. The rate of classical "evaporation" or decay of the time-dependent brane black hole could then be used to place relatively strong bounds on the effective compactification scale, the AdS curvature length $L$ [3]. Later work supporting these ideas appeared in [4, 5].

The key subtlety in the argument of Tanaka and Emparan et al is that the gauge theory is strongly coupled, with large 't Hooft coupling, and hence it is unclear whether simply multiplying the free field result by $N^{2}$ is valid when considering Hawking radiation. The aim of this paper is to revisit this issue with this subtlety in mind.

We find two consistent options for large localized black holes. The first is that static stable solutions exist. The second, although we believe a less likely option, is that static but dynamically unstable solutions exist. The first is obviously in stark contrast to the arguments of Tanaka and Emparan et al. The second, while in detail different - particularly the existence of a static solution, and the interpretation of the instability as being unrelated to Hawking radiation - the qualitative result is similar, namely from the classical 5-d point of view, black holes would shrink at a rate fast enough to be interesting phenomenologically.

We begin by discussing the existence of static black holes in the 5-d classical theory. We use a concrete counter-example to the claim of Tanaka and Emparan et al to argue that in the 4-d holographic dual, Hawking evaporation is not enhanced by a factor of $O\left(N^{2}\right)$, and is therefore absent in the $\hbar \rightarrow 0$ limit. Hence we expect static black holes localized to the brane should exist. We review the likely "pancake" geometry of these localized black holes and interpret it in the 4-d holographic dual as a black hole surrounded by a thermal halo of strongly coupled CFT matter.

We then discuss the issue of dynamical stability of such static solutions. We review using entropy arguments a possible instability suggested by Tanaka that the end of the black hole in the bulk may be unstable to breaking off. Since we expect the localized black holes to have a geometry near the brane which is similar to that of a warped uniform black string, 
this instability should be similar to the type found by Gregory and Laflamme for uniform strings [12]. We explore this mechanism using linear theory to model the possible localized black hole horizon, concluding that this instability is unlikely to occur.

We note that there should be no deep mystery regarding the existence of localized static black holes. In principle their existence is a problem in partial differential equations. Whereas analytically there has been little progress in this [14, 15, 16], numerical methods have been used to solve the full Einstein equations for localized static objects on the brane [17, 18, 19] ${ }^{1}$. The most advanced work is that of Kudoh [21], who indeed finds static localized black holes, but with radii only up to a few AdS lengths. It remains unclear whether large localized black holes exist. We note however that the numerical methods used to find them are presumably difficult to implement for large black holes due to the scale separation between the radius of the horizon and the AdS scale, which must still be resolved. Hence it is unsurprising that very large black holes have not been constructed numerically, and this certainly cannot be taken as evidence against their existence. Which of our above options is realized (stable or unstable static black hole solutions) can only be decisively determined in the non-perturbative gravity theory. It remains interesting to check - and we expect confirm - the stability of black hole solutions by dynamical simulation of the 5 -d classical bulk, using similar methods to [22].

\section{Existence of localized static black holes}

In this section we discuss the validity of the argument of Tanaka and Emparan et al. that no static solution can exist. We use a concrete example of a static black hole in the fivedimensional theory where one can apply their arguments as a counter-example to their claim. We then argue in the holographic dual theory that Hawking radiation is not enhanced by a factor of $O\left(N^{2}\right)$ since the number of asymptotic states that may be radiated is not enhanced by such a factor. Hence we expect a static solution should exist. However, whereas spontaneous Hawking emission to asymptotic states does not occur, there may still be interesting nonspontaneous processes that are seen at the planar level. These might lead to instabilities of this static solution, which we will consider in the section that follows.

\subsection{A counter-example to the claim of no static black holes}

We now present a simple static black hole that falls under the arguments of Tanaka and Emparan et al. Their arguments imply that in the holographic theory a black hole must be spontaneously emitting $O\left(N^{2}\right)$ degrees of freedom, resulting both in the lack of a static solution in the bulk, as one cannot turn off a spontaneous process, and also a deformation of the brane geometry from the usual 4-d Schwarzschild due to the backreaction of this radiation. This example exhibits neither.

The example we consider is the well-known uniform black string. We note that without an IR brane, the bulk geometry is singular, and therefore include one to avoid this subtlety

\footnotetext{
${ }^{1}$ See also 20 .
} 
[23]. Since the metric,

$$
d s^{2}=\left(\frac{L}{z}\right)^{2}\left(g_{\mu \nu}(x) d x^{\mu} d x^{\nu}+d z^{2}\right)
$$

solves the Einstein equations provided $g_{\mu \nu}(x)$ is Ricci flat, we can take $g_{\mu \nu}(x)$ to be the Schwarzschild solution,

$$
g_{\mu \nu}(x) d x^{\mu} d x^{\nu}=-\left(1-\frac{R_{S}}{r}\right) d t^{2}+\left(1-\frac{R_{S}}{r}\right)^{-1} d r^{2}+r^{2} d \Omega^{2}
$$

with horizon radius $R_{S}$ to construct a five-dimensional solution. This solution is the uniformly warped black string. The UV vacuum brane resides at $z=L$, and we introduce a vacuum IR brane at $z=z_{I R}$. While we have included an IR brane, classically we may make $z_{I R}$ as large as we wish. In particular we will take $L<<R_{S}<<z_{I R}$. In this limit the arguments of Tanaka and Emparan et al. would be expected to apply. However, clearly from the form of (2.1), the geometry comprises only a non-trivial zero mode, with no KK modes excited. In particular the UV brane geometry is exactly that of four-dimensional Schwarzschild. Hence from the 4-d dual perspective, the solution is a static Schwarzschild black hole with no backreaction from the CFT.

However, although the static solution exists, this black hole-CFT state is in fact unstable due to the presence of the CFT. For now we continue to focus on the existence of the static black hole solution. We will return to the question of stability in the following section.

\subsection{Reduction of low energy degrees of freedom from strong coupling}

Let us now consider why Tanaka and Emparan et. al.'s calculation of Hawking radiation fails. Tanaka and Emparan et al. [1, 2] proposed a deviation from conventional four-dimensional behavior by considering the four-dimensional holographic interpretation of braneworld black holes. The theory dual to the classical 5-d bulk should be classical 4-d gravity coupled to a large $N$ gauge theory with matter in the 't Hooft planar limit, with 't Hooft coupling $\lambda=N g_{Y M}^{2}$ large, where the gauge theory is conformal in the IR, although the brane acts as a UV cut-off. We will simply refer to the dual theory as a CFT, with this UV cut-off implied. These authors provided evidence that classical bulk geometries correspond in the holographic dual to quantum-corrected black holes. Just as the observer on the brane in the bulk picture sees small deviations from 4-d behavior due to classical 5-d gravity, an observer in the 4-d holographic theory sees the same deviations from classical 4-d behavior, now due to the quantum corrections from planar contributions of the gauge theory.

The 4-d quantum corrections in the holographic dual could survive when we take $\hbar \rightarrow 0$ (note that $\hbar$ is the same for both theories) if quantum effects coherent amongst the $O\left(N^{2}\right)$ color degrees of freedom amount to a total effect going as $\sim N^{2} \hbar$. This should be generally true, and can be seen explicitly for the $\mathcal{N}=4$ case.

Recall that in the large $N$ limit, the 't Hooft coupling

$$
g_{s} N=\left(\frac{L}{l_{s}}\right)^{4}=\lambda
$$


remains fixed, where $g_{s}$ and $l_{s}$ are the bulk string coupling and length. $N$ is related to the 4-d Planck length by $N=L / l_{4}$, so that in this limit $l_{4}=g_{s} l_{s}^{4} / L^{3} \rightarrow 0$. Since $\hbar=l_{4}^{2} / G_{4}$, with $G_{4}$ the 4-d Newton constant, the combined quantity

$$
N^{2} \hbar=\left(\frac{L}{l_{4}}\right)^{2}\left(\frac{l_{4}^{2}}{G_{4}}\right)=\frac{L^{2}}{G_{4}}
$$

remains fixed while $\hbar \rightarrow 0$, as we keep $L, G_{4}$ fixed in RS2.

Consider a 4-d black hole evaporating, with initial radius $R_{S}$. Assume for the moment that as Tanaka and Emparan et al claim, the power emitted by Hawking quanta is $d M / d t \sim$ $N^{2} \hbar / R_{S}^{2}$, ie. $N^{2}$ times the free field result. Then, the evaporation time $T$ remains finite in this $\hbar \rightarrow 0$ limit:

$$
1 / T \equiv \frac{1}{R_{S}} \frac{d R_{S}}{d t}=\frac{1}{R_{S}} \frac{G_{4} d M}{d t}=\frac{L^{2}}{R_{S}^{3}}
$$

We emphasize that this argument holds provided the Hawking radiation rate does indeed go as $N^{2}$ times the usual free field result. We now argue that is not the case.

The problem with the argument is that in the holographic dual theory, there do not necessarily exist $O\left(N^{2}\right)$ dynamical asymptotic degrees of freedom accessible to radiation from a localized finite temperature object. This reduction in accessible degrees of freedom derives from the large 't Hooft coupling when the theory is dual to gravity. ${ }^{2}$

We now briefly review the dynamical degrees of freedom for the usual case of AdS-CFT, where the field theory is $\mathcal{N}=4$ super Yang-Mills, and in particular, how the number of colors $N$ manifests itself in the closed string gravitational dual. Note that although we are using the results for this particular conformal theory, the results should be general whenever the closed string dual has a gravity limit. For details of the review below, the reader is referred to [25, 26, 27, 28, 29, 30, 31, 32] and references therein.

The situation is best understood for the $\mathcal{N}=4$ super Yang-Mills theory on a sphere. Let us take the sphere radius to be $R_{s p h}$. The states in the field theory fall into weakly interacting states with energies $E R_{s p h}<<N^{2}$, and strongly interacting states with energies $E R_{\text {sph }}>>N^{2}$.

The weakly interacting states are built from a free particle Fock basis of traces of products of local adjoint fields and their derivatives. These behave as free particles as in the large $N$ limit these trace operators commute provided the total number of local fields in the product is $<<O\left(N^{2}\right)$, implying an energy $E R_{s p h}<<N^{2}$. These states are best thought of as glueballs, since they represent excitations of the low temperature confining vacuum for the theory on a sphere.

The strongly interacting states arise when the number of local fields in an operator becomes $O\left(N^{2}\right)$, for example considering products of long trace operators or determinants, and hence its energy becomes $E R_{s p h} \sim O\left(N^{2}\right)$. They can no longer be thought of as a set of weakly interacting particles since the commutation with other operators is lost. These states

\footnotetext{
${ }^{2}$ Our argument is related, but different to that in [24] which explained the radiation rate of plasma-balls in large $N$ confining gauge theories.
} 
have large energies, but due to the length of the operators, their density of states is very high, going as $e^{O\left(N^{2}\right)}$. We term these plasma states, since they describe the plasma of the high temperature deconfined phases of the theory on the sphere.

At large 't Hooft coupling the theory is dual to closed string theory in asymptotic AdS in the gravity limit, $\lambda^{1 / 4}=L / l_{s} \rightarrow \infty$. The glueball states should then correspond to perturbative string excitations about the vacuum target spacetime. While in the free theory the separation of energies of the glueball states is $\sim 1 / R_{s p h}$, at large 't Hooft coupling the energy separation scales as $\sim \lambda^{1 / 4} / R_{\text {sph }}$ since the spacing of the closed string dual spectrum is $\sim 1 / l_{s}$ rather than $\sim 1 / L$. Hence as we approach the gravity limit the entire glueball spectrum is lifted to infinite energy, apart from the glueballs dual to the supergravity modes of the string. Then the gravitational perturbations are dual to only $O(1)$ of the $O\left(N^{2}\right)$ free particle basis states. In particular the graviton is dual to the field theory stress tensor. We cannot see ' $\mathrm{N}$ ' for perturbative fluctuations in the gravity limit, the other $O\left(N^{2}\right)$ perturbative states only becoming visible when we look at string scale physics.

In our situation we do not restrict the field theory to be $\mathcal{N}=4$ super Yang-Mills, the case discussed above. However, in other field theories known to be dual to string theories the case is qualitatively similar [30]. Since in these cases the reduction in light glueball degrees of freedom is exactly dual to the decoupling of string oscillator modes in the string dual when truncating to the gravity limit, it seems reasonable that this will occur whenever the string dual to the field theory has such a gravity truncation.

At large 't Hooft coupling the plasma states correspond to the non-perturbative black hole excitations in the closed string dual, the small and large AdS Schwarzschild black holes with radii small or large compared to the AdS scale $L$. The energies of these black holes are $E R_{s p h} \sim O\left(N^{2}\right)$ translated to the field theory. The large number density of the plasma states allows them to account for the $O\left(N^{2}\right)$ entropy of these black holes. At finite temperature, whilst at large $N$ these states are very massive, their enormous number density, $e^{O\left(N^{2}\right)}$, may allow them to overcome their exponential Boltzmann suppression. This occurs for the large black holes which are rapidly semiclassically spontaneously nucleated in hot AdS above a critical temperature in the field theory going as $\sim 1 / R_{s p h}$, and is reflected in the gravity dual by the negative free energy of the large black holes. The small black holes, similar to asymptotic flat space black holes have positive free energy, and hence are composed of states that are not numerous enough to spontaneously overcome their Boltzmann suppression.

Having briefly reviewed how field theory states correspond to the dual gravity physics, and in particular the role that the number of colors $N$ plays, we now consider radiation from a localized finite temperature object coupled to the CFT at strong coupling in asymptotic flat space, such as for our 4-d black hole localized to the brane. We again are interested in the $\lambda \rightarrow \infty$ limit to ensure the gravity dual description, and may still consider the theory on the sphere although we must take the sphere radius $R_{s p h}$ to be much larger than the size of our localized thermal source, the black hole.

Firstly consider the glueball excitations. The $O(1)$ free particle states dual to the graviton perturbation modes, which have energies $\sim 1 / R_{s p h}$, can certainly be radiated. However as 
described above the remainder of the $O\left(N^{2}\right)$ glueball states cannot, due to their enormous energy $\sim \lambda^{1 / 4} / R_{s p h}$, and hence enormous Boltzmann suppression. In the gravity dual, this corresponds to our localized black hole thermally radiating gravitons, but not effectively radiating string oscillator modes due to their enormous mass.

Secondly we must consider the plasma states. The only way these can be emitted spontaneously is if they have a sufficiently large degeneracy to overcome their large $O\left(N^{2}\right)$ energy, and hence large Boltzmann suppression. In principle this might allow an emission rate that could account for a classical process in the dual gravity.

Whilst a thermal emission of such massive objects naively seems unlikely (see [33] for a discussion of Hawking radiation of 'macroscopic' objects), and our previous counter-example of the warped string demonstrates explicitly that this does not occur, we now attempt to give a rough argument why this is so.

We expect that the plasma states dual to small black holes cannot be spontaneously nucleated by our localized black hole, since they cannot even be nucleated when the entire theory is put at finite temperature. So we only consider the possible nucleation of plasma states dual to large black holes with radius of order $L$ or greater.

Consider now in the bulk the large black holes that might be 'classically emitted' from the localized brane black hole or black string of the counter-example. Since we are interested in the Poincare slicing of AdS, there are no finite size static black holes (not attached to the brane). The only static black holes are infinite in extent in the brane directions, and hence have infinite energy, and correspond to a horizon at finite radial position in the bulk. Obviously such infinite energy objects could not be radiated by a finite size hot source such as our localized black hole or string.

However, we must also be concerned with black holes of large but finite radius that are classically emitted near the brane black hole horizon, and then subsequently fall away from the brane. Such black holes have a temperature measured on the brane given by their local horizon temperature, redshifted by the warp factor due to their distance from the brane. Hence the temperature of a black hole decreases as it falls away from the brane, which is dual to the temperature of the thermal plasma decreasing as it expands under its internal pressure.

Static large black holes in global AdS have a temperature that increases with their energy. There is a minimum black hole temperature, attained by a black hole of approximately $L$ in radius. Such a black hole of radius $L$, within a few AdS lengths of the brane will then have a temperature measured on the brane given as $\sim 1 / L$. Taking this minimum temperature large black hole and treating it as a probe in the AdS metric written as (2.1), we can estimate its temperature reduced by the redshifting at its coordinate distance $z$ from the brane as $T \sim 1 / z$.

The important point is that the brane black holes (localized or string) have lower brane temperature the larger their radius $R_{S}$, going as $T \sim 1 / R_{S}$ for $R_{S}>>L$. Since a thermal object cannot emit objects hotter than itself, a large 4-d black hole evidently cannot emit plasma states near its horizon dual to this minimum temperature black hole within the region 
$z<R_{S}$ near the brane. This remains true for plasma states dual to even larger black holes, which have even larger temperatures. Now we should consider the plasma states dual to the minimum temperature large black hole far from the brane. For $z>R_{S}$ the brane temperature of these black holes becomes small enough that the dual plasma states might be emitted. However, for the string the warp factor means the local horizon radius of the string far from the brane will be much smaller than $L$, and hence there is little overlap of the string and the large black hole of radius $\sim L$ to be emitted, and hence such a process would be suppressed. For the localized brane black hole, as we shall see later the horizon doesn't extend further than $z \sim R_{S}$ into the bulk, and hence again there is a lack of overlap which would disallow the process. We therefore conclude that the only plasma states that have any chance to be emitted are those that are dual to black holes with radius $\sim L$, at a position $z \sim R_{S}$ in the bulk. We have no argument to rule these out, but our counter-example implies that the effects ruling out the emission of both the $z<R_{S}$ and $z>R_{S}$ black holes are still sufficiently effective at $z \sim R_{S}$ to stop emission there too.

Since we cannot solve the CFT at large 't Hooft coupling the above arguments are necessarily heuristic. However, they do show how the simple thinking that one computes the Hawking radiation rate by multiplying the free field result by $O\left(N^{2}\right)$ breaks down in the strongly coupled field theory dual to gravity. We therefore conclude there is no convincing holographic argument obstructing the existence of 5-d static black holes localized on the brane due to spontaneous radiation in the field theory. Furthermore we do not expect any backreaction from this field theory Hawking radiation to be seen in the classical gravity dual either. This is in perfect accord with the static warped uniform string example given above where neither classical radiation or its backreaction is seen. However the situation is interesting when one considers dynamical stability of static solutions. In the following section we will consider possible classical instabilities and why instabilities, but not spontaneous Hawking radiation, might survive in the large $N$ planar limit.

Notice that we have done the analysis in the AdS background that is relevant to phenomenological applications. It is interesting to note that whereas the localized black hole appears to exist without spontaneously radiating in a cold vacuum, if we heat the theory up to temperatures of order the localized black hole temperature or higher, this metastability is likely to be effected. In this case, the holographic picture indicates that the localized black hole should radiate strongly, at a rate $O\left(N^{2}\right)$, since the relevant degrees of freedom at this high temperature are deconfined with all $O\left(N^{2}\right)$ gluons contributing [28], so it may evaporate in line with the original arguments of Tanaka and Emparan et al. This radiation would be due to the emission of the non-perturbative plasma states of the thermal bath, whose high temperature 'evaporates' the localized hot object. From the 5-d perspective, we note that the gravity dual description now includes an IR horizon in the vicinity of the brane that likely disallows the static localized 5-d horizon on the brane. In this case, the brane black hole horizon ends on the bulk black hole and thus, instead of rounding off at the tip, looks stringlike everywhere. This eliminates the need for the CFT modes dual to the "rounding-off" behavior, since the black string is described purely by gravity in the CFT. Of course, putting 
the 4-d theory in such a high temperature thermal plasma bath is interesting but not a case relevant for phenomenology and we will not discuss this further here.

\subsection{Geometry of localized black holes}

As we now know of no argument, holographic or otherwise ${ }^{3}$, that a static localized black hole should not exist, we review what form they are expected to take. Following Giddings et al [8], we may estimate the shape of a black hole from the linear equation governing the field $\phi=1+\left(z^{2} / L^{2}\right) g_{00}$, i.e. the AdS scalar Laplacian. We estimate the intrinsic horizon spatial geometry as that induced on the isosurface where $\phi=1$. Of course it is unclear how accurate the linear approximation will be to the full non-linear solutions, but it is reasonable to expect qualitative agreement.

Taking the AdS coordinates,

$$
d s^{2}=\frac{L^{2}}{z^{2}}\left(-d t^{2}+d r^{2}+r^{2} d \Omega^{2}+d z^{2}\right)
$$

the AdS Laplacian is homogeneous in $r$ and $z$,

$$
\square \phi=\left(\frac{z}{L}\right)^{2}\left(\partial_{r}^{2}+\frac{2}{r} \partial_{r}+\partial_{z}^{2}-\frac{3}{z} \partial_{z}\right) \phi
$$

Taking the brane to be at $z=L$, we must solve the Laplace equation for Neumann boundary conditions at the brane, but with a static delta function source at $r=0$. Following Giddings et al, one then constructs the metric perturbation from $\phi$. The strength of the source determines the size of the black holes, and hence the position of the locus $\phi=1$. The brane and delta function position being at $z=L$ breaks the homogeneous scaling symmetry of the equation and other boundary conditions, $r, z \rightarrow \lambda r, \lambda z$ under a change in strength $\lambda^{2}$ of the delta function source. However, far from the brane compared to the AdS scale, the solution does regain this scaling symmetry. Hence for large black holes, so that the majority of the isosurface $\phi=1$ is many AdS lengths from the brane, the horizon isosurfaces have the same shape in the $r, z$ plane, up to the global scaling of $r$ and $z$. This is illustrated in figure 1 where we plot isosurfaces for a variety of black hole sizes and we see the larger black holes all have the same shape. The horizon isosurface extends a coordinate distance approximately $\Delta z \sim 2 R_{S}$ into the bulk.

The shape of the isosurface in the $r, z$ plane implies that for large black holes with horizon radius $R_{S}>>L$ on the brane, the horizon geometry near the brane will be approximately that of a warped uniform string extending into the bulk. Around a proper distance $\simeq L \log R_{S} / L$

\footnotetext{
${ }^{3}$ The work of Bruni et al [34 showed a collapsing shell of matter which is spherically symmetric on the brane cannot have a static Schwarzschild exterior unlike in conventional 4-d gravity. This is often cited as supporting the conjecture that no static localized black hole exists. Since Birkhoff's theorem no longer applies outside a spherical brane matter source, due to the presence of the KK modes, it is entirely to be expected that the exterior brane metric is not static, while the spherically symmetric KK modes get radiated away. Of course the geometry will settle down to the static localized black hole eventually, in no contradiction with the results of 34.
} 


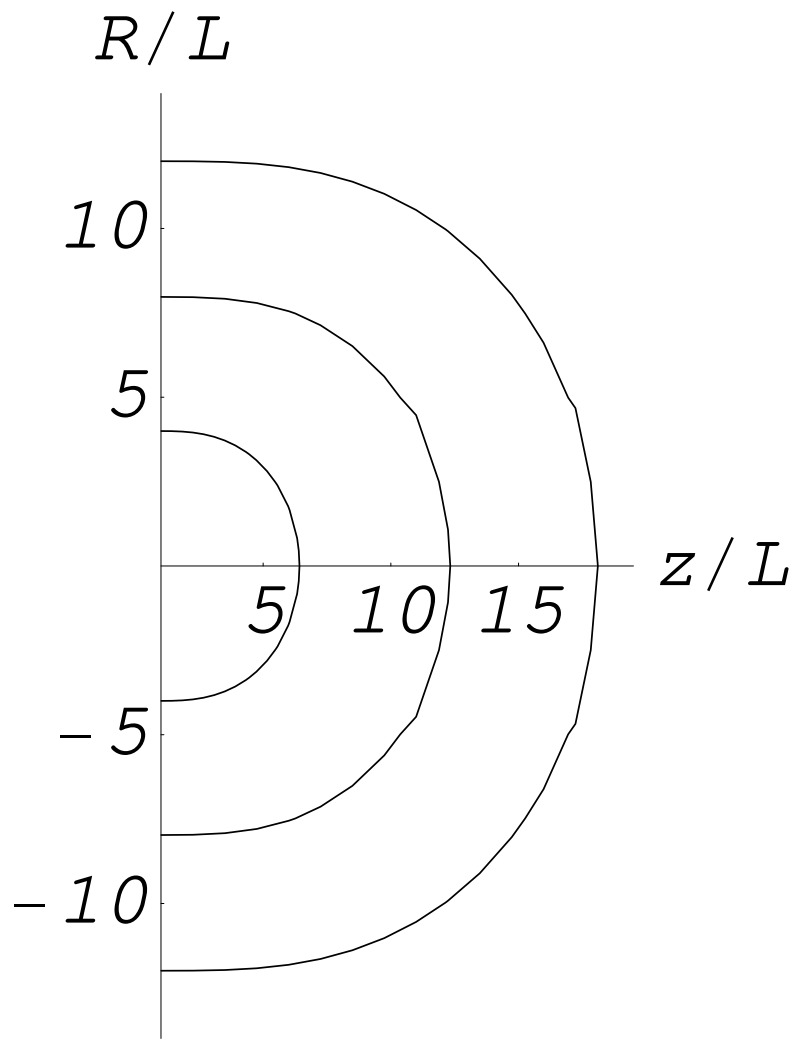

Figure 1: Surfaces in AdS in $r, z$ coordinates, computed from linear theory, whose intrinsic geometry approximates the horizon geometry of black holes of 3 sizes: $4 L, 8 L$, and $12 L$. We see these large localized black holes have horizon geometries simply related by a global scaling, and extend a coordinate distance $\Delta z \sim 2 R_{S}$ into the bulk.

this warped uniform string ends, being capped off in an additional proper distance $\sim L$ by a horizon with characteristic curvature radius $\sim L$. Since this capping off necessarily involves nonzero mass Kaluza-Klein modes, in the 4-d holographic theory, the black hole horizon will be surrounded by a strongly coupled halo of gauge theory matter bound to it. We expect that 
only glueballs dual to gravitons may be spontaneously radiated from its surface in the planar limit. The interesting question is then whether this black hole and halo state is dynamically stable.

The possible existence of black hole solutions with bound CFT matter might initially seem surprising. Nonetheless, we know that such bound states exist in other situations, the simplest example being localized matter on the brane, such as stars and planets. Similarly, extremal black holes should take this form. Such solutions perturb the bulk geometry by sourcing Kaluza-Klein modes, and therefore have a bound state CFT component from a holographic perspective.

\section{Stability of localized static black holes}

We have shown a concrete example of a static black hole in a 4-d theory of gravity plus CFT where the Tanaka and Emparan et al arguments would predict none could exist. However in this theory, as we discuss below, this exactly Schwarzschild black hole plus trivial CFT state is actually unstable, and thus may have interesting dynamics in the spirit of their arguments. Having concluded that static black holes exist, we now discuss their dynamical stability.

There are two possibilities. The first is that there is a consistent stable black hole bound state with the CFT. The second is that a solution exists but is unstable. A third possibility, that no static localized black hole solution exists, is not ruled out by our counter-example to the arguments of Emparan et al., though it does remove the argument against their existence. However, we re-emphasize that static black hole solutions up to a few AdS lengths have been numerically constructed, so that static (though perhaps unstable) solutions should exist. Interestingly, if an instability were present for the localized black hole, the end result of such a process may be rather similar to Tanaka and Emparan et al's picture, namely a rapid loss of energy to infinity, analogous to the result of the Gregory-Laflamme instability. We discuss the form such an instability might take for the brane black hole shortly. We now briefly discuss the time-dependent evolution that would occur in the presence of such an instability.

Emparan et. al. suggested that the bulk interpretation of black hole decay would be classical gravitational radiation near the brane, through which the black hole slides off the brane into the bulk. However, this interpretation is clearly problematic in that the only light mode localized near the brane is the zero mode, and that mode (in RS2) is not a CFT bound state, but a fundamental normalizable mode that exists in the presence of the brane. Since any potential instability should be a consequence of the CFT dynamics, the bulk holographic interpretation must lie elsewhere.

Tanaka [1] made a different suggestion which is more likely. The black hole could decay classically through emission of higher-dimensional black holes at the tip, where the curvature is large - of size set by the AdS scale, the size of the tip region. Specifically, the tip of the black hole, where it extends farthest away from the brane, is unstable to breaking off. The remaining brane black hole would become slightly smaller, and the tip would become a small higher-dimensional black hole which would then decay or falls through the horizon of the 
Poincaré patch. From the CFT point of view, this would correspond to an instability through which the black hole could decay much more rapidly than implied by Hawking radiation.

Tanaka estimated the rate at which the brane black hole would lose energy to the small black droplets. The total rate of energy released is the mass of the black droplets multiplied by the rate of droplet production. Although we cannot give a precise rate, from the perspective of the local 5-d geometry, there is only one scale in the problem, $L$. The only other scale, $R_{S}$ the horizon radius, does not appear locally. It seems reasonable to assume that the rate of black hole production is $L^{-1}$. The total rate of energy production is then

$$
\frac{d E_{t o t}}{d t} \sim\left(M_{d r o p}\right) \frac{d N}{d t}=\left(L^{2} M_{5}^{3}\right)\left(L^{-1}\right)
$$

with $M_{5}$ the 5-d Planck mass. An observer on the brane will see this value redshifted by the factor $\left(L / R_{S}\right)^{2}$, so the observed evaporation rate will be

$$
\frac{d E_{\text {obs }}}{d t} \sim \frac{L^{3} M_{5}^{3}}{R_{S}^{2}}=N^{2} A T^{4}
$$

where we have used the holographic relation $N^{2}=M_{5}^{3} L^{3}$. We have written the last expression in terms of the 4 -d parameters, the area $A$ of the 4 -d black hole and its temperature $T=1 / R_{S}$. This gives parametrically the same rate of energy loss in the CFT as Tanaka and Emparan et al's proposal of spontaneous thermal emission of $O\left(N^{2}\right)$ degrees of freedom. However we note that in light of the arguments in the previous section, this is not a spontaneous process, but rather an instability, and hence can be turned off by fine tuning.

\subsection{Gregory-Laflamme instability}

We now consider the warped black string instability. Afterward we will consider a possible analogous instability for the black hole. The known instability (for the black string) is the Gregory-Laflamme (GL) instability [12, 35, 13], which we now review. Consider disturbing the warped uniform string metric (2.1) by a 4 -d tensor perturbation,

$$
d s^{2} \rightarrow d s^{2}+h_{\mu \nu}(t, r, z) d x^{\mu} d x^{\nu}
$$

Gregory showed [35] that the usual vacuum GL instability of uniform strings generalizes simply to the warped case. This was not at all obvious as the warped string background is not translationally invariant. Writing,

$$
h_{\mu \nu}(t, r, z)=\chi_{\mu \nu}(t, r) f(z)
$$

we take the 4-d tensor $\chi_{\mu \nu}$ to be transverse and traceless with respect to the 4 -d metric $g_{\mu \nu}(x)$. Taking $f(z)$ to be an eigenmode of the operator, $\partial_{z}^{2}-\frac{3}{z} \partial_{z}$, with eigenvalue $k^{2}$, so that,

$$
f(z)=\mathcal{A} J_{2}(k z)+\mathcal{B} N_{2}(k z)
$$


and we must choose coefficients $\mathcal{A}, \mathcal{B}$ to satisfy the appropriate boundary conditions. Then the 4-d tensor perturbation $\chi_{\mu \nu}$ satisfies,

$$
\left(\triangle_{L}^{(4)}+k^{2}\right) \chi_{\mu \nu}=0
$$

where $\triangle_{L}^{(4)}$ is the Lichnerowicz operator of the 4-d metric $g_{\mu \nu}$ given by,

$$
\triangle_{L}^{(4)} \chi_{\mu \nu}=\nabla_{(4, S)}^{2} \chi_{\mu \nu}+2 R_{\mu \nu}^{(4) \alpha \beta} \chi_{\alpha \beta}=0
$$

with $\nabla_{(4, S)}^{2}$ the 4-d metric scalar Laplacian, and $R_{\alpha \beta \mu \nu}^{(4)}$ the 4-d metric curvature, with indices raised and lowered with respect to the 4-d metric $g_{\mu \nu}$.

This is exactly the equation one obtains for a non-warped string. Hence as in that case, for $k<k_{c}=0.45 / R_{S}$ one finds modes with an exponentially growing $t$ dependence, leading to the familiar horizon instability. If we consider an IR brane, then the spectrum of allowed $\lambda$ becomes quantized, although provided that $z_{I R}>>R_{S}$, these include unstable modes.

\subsection{Holographic interpretation of instabilities}

We see from the warped uniform string example that the CFT state may be dynamically unstable. There is no contradiction with our statements about Hawking radiation, since this instability is not a spontaneous radiative process, but rather is simply a result of having an energetic instability. The GL instability of gravity implies the existence of certain tachyonic modes in the gauge theory description. In the gravity these modes are perturbative graviton states, and hence in the gauge theory correspond to tachyonic glueballs. Note that these tachyonic glueball states are quite specific in form, being spherically symmetric, and exponentially localized near the horizon. This instability is not spontaneous, as by suitable fine tuning the system can be prepared to stay static for as long as we wish. However, when perturbed the occupation number in these tachyonic glueball modes will grow simply because it is energetically favorable for this to happen. This will continue until a large collective behavior is produced. Thus we conclude that dynamical instabilities of the CFT vacuum may lead to interesting dynamics in the planar limit, but spontaneous radiation is not seen in this limit. The Emparan et al argument fails for spontaneous emission of radiation, but interesting effects analogous to their original claims might be possible for non-spontaneous energetic reasons.

\subsection{A possible instability}

So let us now consider the possibility of a GL-like instability for the localized brane black hole in the bulk. The majority of the proper distance of a large localized black hole with brane radius $R_{S}>>L$ appears like the warped uniform string, extending roughly $L \log R_{S} / L$ into the bulk, and it is only in the last $L$ proper distance that the geometry deviates from the uniform string and caps off.

The GL instability is the only dynamical instability known for static black holes, and certainly would account for the decay of the black hole that was considered above. Gregory 
and Laflamme argued that for a uniform string the natural end state of the instability in vacuum is an array of black holes. This remains an issue of controversy due to the result of Horowitz and Maeda [36]. However, following numerical simulation of the instability by Choptuik et al [22], it has recently been argued that Gregory and Laflamme's original picture is likely to be correct [37, 38, 39]. If the uniform string region of a localized black hole were long enough, one would expect a similar instability to exist on it, whose dynamics would result in the uniform neck breaking up, and the segments not connected to the brane falling into the bulk.

In the CFT such an instability would manifest itself through the existence of tachyonic glueball states. Perturbing the static black hole and its halo would result in a condensation into these tachyonic states. The result would be violent emission of glueballs (corresponding to gravity waves in the 5-d gravity), and expanding cooling shells of thermal gluon plasma (corresponding to the small black holes falling away from the brane).

For a large black hole, the profile of the GL instability, given by $f(z)$ in equation (3.4) goes as,

$$
f(z) \sim J_{2}(k z)
$$

where we recall that the marginally unstable mode has eigenvalue $k_{c}=0.45 / R_{S}$. We plot this profile in figure 2. Earlier we estimated the shape of a large black hole using the linear theory, finding that the coordinate extent, $\Delta z$, of the potential isosurface we take to approximate the horizon into the bulk is $\Delta z=2 R_{S}$. We also plot this in figure 2. We might take the region of the isosurface extending a half or quarter of this distance to approximate a uniform warped string. However, from figure 2, it is then clear that only a tiny fraction of a wavelength of the marginal mode could fit into this region. Hence we find it very unlikely that any potential Gregory-Laflamme instability could be localized in the uniform string region of the localized black hole.

\subsection{Entropy Balance}

Even without finding the explicit black string instability, one could have argued via entropy considerations that one would expect that the black string is unstable. We now apply this reasoning to the brane black hole by comparing the entropy advantage of the localized black hole to "drip" off a small black hole at the tip to the entropy of the static solution. We find that from the perspective of entropy considerations, the decay is parametrically marginal, so it cannot be decided according to entropy considerations.

Consider a brane black hole that extends into the bulk out to some value $z$ in $z$-coordinates which is close to $R_{S}$. If the tip were to drip off, then it would form a small black droplet of radius $L$, and the brane black hole would shrink. The black droplet will have mass and entropy given approximately by

$$
\begin{aligned}
\delta M_{\text {drop }} & \sim M_{5}^{3} L^{2} \\
\delta S_{\text {drop }} & \sim M_{5}^{3} L^{3}
\end{aligned}
$$




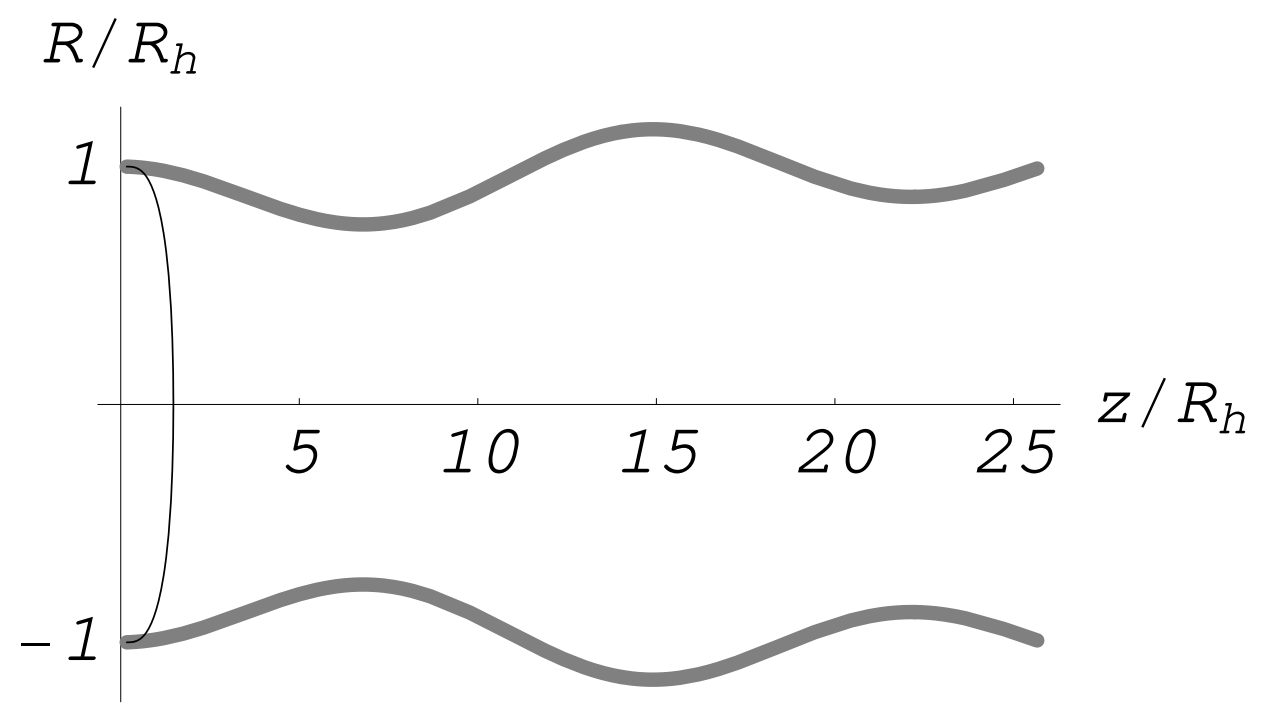

Figure 2: The horizon of the brane black hole from linearized gravity and the horizon of the perturbed black string. The perturbation shown is the unstable mode with the shortest wavelength.

The brane black hole will shrink by an amount given by energy conservation. The effective mass lost to the black droplet is redshifted by the warp factor:

$$
\delta M_{b b h}=-\delta M_{d r o p} \frac{L}{z}
$$

The radius of the brane black hole then shifts by an amount $\delta R_{S}=\delta M_{b b h} / M_{4}^{2}$, and the entropy shifts by

$$
\delta S_{b b h} \approx M_{5}^{3} L\left[\left(R_{S}-\delta R_{S}\right)^{2}-R_{S}^{2}\right] \sim-M_{5}^{3} L^{3} \frac{R_{S}}{z}
$$

For $z \approx R_{S}$, this is parametrically the same as the entropy of the black droplet. However, if the brane black hole sticks farther out into the bulk, then the total entropy change $\delta S_{d r o p}+\delta S_{b b h}$ grows and becomes positive, and formation of a black droplet is favored.

Notice that for $z<R_{S}$, this analysis wouldn't apply since the black holes that would be spit off in that case would be larger than the AdS length scale, so the simple interpretation as pure five-dimensional flat space black holes would certainly not apply. The analysis is only valid up to the point where the entropy argument is indeterminate.

The formula for the entropy $S_{b b h}$ of the brane black hole deserves a quick comment. The black hole extends a proper distance into the bulk, so a naive estimate of the area would be $S_{b b h} \sim R_{S}^{2} L \log R_{S} / L$. However, as the authors of [40] discuss, the contribution to the area is suppressed by the warp factor away from the brane, and almost all of the area is near the brane itself. A better estimate for the area is

$$
d \mathcal{A}=\frac{4 \pi R_{S}^{2} d z}{(z / L)^{3}}
$$


on each spatial slice of constant $z$. Integrating $d \mathcal{A}$ from $z=L$ to $z=R_{S}$ accounts for the formula for $S_{b b h}$.

As there is no parametric argument for, or against this instability we regard it as marginal. We expect that any modification of the bulk physics might therefore effect this stability. For example, adding charges to a uniform black string may render the GL instability absent [41]. Certainly extremally charged localized black holes will be stable.

Another simple modification is to add extra compact dimensions. In this case, the area of the small black droplet can start to probe the extra dimensions, whereas the large brane black hole will fill them up. Consider for example a brane black hole in $5+D$ dimensions where $D$ of the dimensions are compact and of size $L$. The surface area of a unit $3+D$-sphere is smaller than that of a 3 -sphere by the factor

$$
\frac{\int d \Omega_{3+D}}{\int d \Omega_{3}}=\left(\frac{(2 \pi)^{D / 2}}{3 \cdot 5 \cdots(3+D-2)}\right)
$$

Since the black droplet in this scenario is roughly the same size as the compact dimensions, it will interpolate between a $4+1$-dimensional black hole and a $4+D+1$ dimensional black hole, and its entropy will pick up some fraction of the above ratio. By making $D$ large, it becomes more likely that such the brane black hole is stable.

\section{Effective Theory Interpretation}

We now return to the issue mentioned in the introduction of why the effective theory for linear fluctuations about a flat brane can be given by pure 4-d gravity, but even for large radius black holes this 4-d gravity description can break down. We note that this is true whether or not the black hole is unstable. In either case, the effective theory at the nonlinear level in the presence of a black hole breaks down.

This might seem surprising since for the linear theory about the flat brane we recover simple 4-d gravity for perturbations with wavelength larger than $L$, the AdS length. Thus one might naively assume that 4-d gravity is the correct effective theory with a cut-off requiring all curvature radii to be less than $L$. However, this is not the case. Since the Kaluza-Klein spectrum is gapless, when we consider a background containing a black holes with radius $R_{S}$ we should worry about integrating out the modes with masses $m$ less than $\sim 1 / R_{S}$. Usually theories that admit 4-d effective descriptions have a mass gap in their spectrum and hence for large enough black holes there will be no modes with such masses. Here however there is a continuum of such light modes, down to zero mass. While the spectrum of large mass modes, with masses $m>>1 / R_{S}$ will be unchanged due to the presence of the black hole in the background, the spectrum of the modes with masses approximately $m \sim 1 / R_{S}$ will generically be strongly affected.

This modification of the effective theory is important and perhaps more obvious when considering the quantum radiation rate of the 5-d black hole. From a purely 5 -d perspective, there is only a single graviton with $\mathcal{O}(1)$ degrees of freedom leading to a relatively small 
radiation rate 42]. However, if one were to calculate the decay rate using the $4 \mathrm{~d}$ theory with the original modes, one would find that the answer would depend on an IR and a UV cut-off. The resolution of this apparent discrepancy is that the black hole has drastically changed the spectrum of KK modes. The picture is qualitatively the same as the usual calculation for Schwarzschild black holes. The phase space of graviton excitations in flat space gets replaced in the presence of the black hole by spherical harmonics, which are effectively quantized at the temperature of the black hole, and oscillations in the radial direction. In the case of the localized black hole, the KK modes no longer have a continuous spectrum, but effectively are quantized also at the temperature of the black hole, leaving only $\mathcal{O}(1)$ accessible modes. So in general, an effective theory with a KK spectrum continuous down to zero can change in the presence of large geometric perturbations such as a black hole. Note, however, that in the case of the brane black hole, modes with mass lighter than the temperature have small overlap with the higher-curvature region of the brane black hole. Therefore only those modes with mass of order the black hole temperature would be strongly coupled.

Consider again our simple example of the warped uniform string from section 2.1. In the dual 4-d theory this does appear to be exactly a 4-d Schwarzschild solution. However, as we have discussed above, the Kaluza-Klein spectrum about this solution has tachyonic modes, due to the GL instability. These are not present in the spectrum of fluctuations in the absence of the black hole, and only arise only through the non-linear interaction of the Kaluza-Klein modes with the 4-d graviton. We see this non-linear interaction explicitly in equation (3.3), where the perturbation governed by $\chi_{\mu \nu} f(z)$ is a Kaluza-Klein mode, and the Lichnerowicz operator includes the non-linear coupling to the 4-d zero mode, $g_{\mu \nu}(x)$, through $R_{\mu \nu}^{(4) \alpha \beta} \chi_{\alpha \beta}$ acting as a potential. Since the mass squared, $-k^{2}$, may become arbitrarily small, when it becomes of order the potential $\sim 1 / R_{S}^{2}$, the 4 -d mode behavior is strongly modified from that of a usual 4-d field with the same mass.

Note that in addition to the $4 \mathrm{~d}$ coupling of the graviton to the tower of KK modes, higher dimensional operators become more important because of the large curvature components at the tip. For both these reasons, conventional no-hair theorems do not apply. Hence there can be two different types of black hole solutions - one involving KK modes (the brane black hole we have been discussing) and one without them (the black string).

\section{Acknowledgments}

We would like to thank Nima Arkani-Hamed, Roberto Emparan, Hideaki Kudoh, Lubos Motl, Robert Myers, and Veronica Sanz for very interesting discussions. T.W. is grateful for the stimulating environment and hospitality provided at the KITP workshop "Scanning New Horizons: GR Beyond 4 Dimensions". The research of T.W. is supported by NSF grant PHY0244821. L.R. and A.L.F. are supported by NSF grants PHY-0201124 and PHY-0556111, and A.L.F. acknowledges an NSF graduate research fellowship. 


\section{References}

[1] T. Tanaka, "Classical black hole evaporation in Randall-Sundrum infinite braneworld," Prog. Theor. Phys. Suppl. 148 (2003) 307 [arXiv:gr-qc/0203082].

[2] R. Emparan, A. Fabbri and N. Kaloper, "Quantum black holes as holograms in AdS braneworlds," JHEP 0208, 043 (2002) [arXiv:hep-th/0206155].

[3] R. Emparan, J. Garcia-Bellido and N. Kaloper, "Black hole astrophysics in AdS braneworlds," JHEP 0301, 079 (2003) [arXiv:hep-th/0212132].

[4] P. R. Anderson, R. Balbinot and A. Fabbri, "Cutoff AdS/CFT duality and the quest for braneworld black holes," Phys. Rev. Lett. 94, 061301 (2005) [arXiv:hep-th/0410034].

[5] R. Casadio and C. Germani, "Gravitational collapse and black hole evolution: Do holographic black holes eventually 'anti-evaporate'?," Prog. Theor. Phys. 114, 23 (2005) [arXiv:hep-th/0407191].

[6] H. Verlinde, "Holography and compactification," Nucl. Phys. B580, 264 (2000) [arXiv:hep-th/9906182].

[7] S. Gubser, "AdS/CFT and gravity," Phys. Rev. D63, 084017 (2001) [arXiv:hep-th/9912001].

[8] S. B. Giddings, E. Katz and L. Randall, "Linearized gravity in brane backgrounds," JHEP 0003, 023 (2000) [arXiv:hep-th/0002091].

[9] M. Duff and J. Liu, "Complementarity of the Maldacena and Randall-Sundrum pictures," Phys. Rev. Lett. 85, 2052 (2000) [arXiv:hep-th/0003237].

[10] S. Giddings and E. Katz, "Effective theories and black hole production in warped compactifications," J. Math. Phys. 42, 3082 (2001) [arXiv:hep-th/0009176].

[11] N. Arkani-Hamed, M. Porrati and L. Randall, "Holography and phenomenology," JHEP 08, 17 (2001) [arXiv:hep-th/0012148].

[12] R. Gregory and R. Laflamme, "Black strings and p-branes are unstable," Phys. Rev. Lett. 70 (1993) 2837 [arXiv:hep-th/9301052].

[13] R. Gregory and R. Laflamme, "The Instability of charged black strings and p-branes," Nucl. Phys. B 428, 399 (1994) [arXiv:hep-th/9404071].

[14] R. Emparan and H. Reall, "Generalized Weyl solutions," Phys. Rev. D65, 084025 (2002) [arXiv:hep-th/0110258]

[15] C. Charmousis and R. Gregory, "Axisymmetric metrics in arbitrary dimensions," Class. Quant. Grav. 21527 (2004) [arXiv:gr-qc/0306069]

[16] S. Creek, R. Gregory, P. Kanti and B. Mistry, "Braneworld stars and black holes," arXiv:hep-th/0606006.

[17] T. Wiseman, "Relativistic stars in Randall-Sundrum gravity," Phys. Rev. D65 124007 (2002) [arXiv:hep-th/0111057].

[18] A. Chamblin, H. Reall, H. Shinkai and T. Shirmizu, "Charged brane-world black holes," Phys. Rev. D63 064015 (2001) [arXiv:hep-th/0008177]. 
[19] H. Kudoh, T. Tanaka and T. Nakamura, "Small localized black holes in braneworld: Formulation and numerical method," Phys. Rev. D68 024035 (2003) [arXiv:gr-qc/0301089].

[20] R. Casadio and L. Mazzacurati, "Bulk shape of brane-world black holes," Mod. Phys. Lett. A 18, 651 (2003) [arXiv:gr-qc/0205129].

[21] H. Kudoh, "6-dimensional localized black holes: Numerical solutions," Phys. Rev. bf D69 104019 (2004) [arXiv:hep-th/0401229].

[22] M. Choptuik et al, "Towards the final fate of an unstable black string," Phys. Rev. D68 044001 (2003) [arXiv:gr-qc/0304085].

[23] A. Chamblin, S. W. Hawking and H. S. Reall, "Brane-world black holes," Phys. Rev. D 61, 065007 (2000) [arXiv:hep-th/9909205].

[24] O. Aharony, S. Minwalla and T. Wiseman, "Plasma-balls in large N gauge theories and localized black holes," Class. Quant. Grav. 232171 (2006) [arXiv:hep-th/0507219].

[25] M. Maldacena, "The large N limit of superconformal field theories and supergravity," Adv. Theor. Math. Phys. 2231 (1998) [arXiv:hep-th/9711200].

[26] E. Witten, "Anti-de Sitter space and holography," Adv. Theor. Math. Phys. 2235 (1998) [arXiv:hep-th/9802150].

[27] S. Gubser, I. Klebanov and A. Polyakov, "Gauge theory correlators from non-critical string theory," Phys. Lett. B428 105 (1998) [arXiv:hep-th/9802109].

[28] E. Witten, "Anti-de Sitter space, thermal phase transition, and confinement in gauge theories," Adv. Theor. Math. Phys. 2505 (1998) [arXiv:hep-th/9803131].

[29] O. Aharony, S. Gubser, J. Maldacena, H. Ooguri and Y. Oz, "Large N field theories, string theory and gravity," Phys. Rept. 323183 (2000) [arXiv:hep-th/9905111].

[30] M. Strassler and J. Polchinski, "Deep inelastic scattering and gauge/string duality," JHEP 0305012 (2003) [arXiv:hep-th/0209211].

[31] B. Sundborg, "The Hadgedorn transition, deconfinement and N = 4 SYM theory," Nucl. Phys. B573 349 (2000) [arXiv:hep-th/9908001]

[32] O. Aharony, J. Marsano, S. Minwalla, K. Papadodimas and M. Van Raamsdonk, "The Hagedorn / deconfinement phase transition in weakly coupled large N gauge theories," Adv. Theor. Math. Phys. 8, 603 (2004) [arXiv:hep-th/0310285].

[33] D. Marolf and R. Sorkin, "On the status of highly entropic objects," Phys. Rev. D29, 024014 (2004) [arXiv:hep-th/0309218].

[34] M. Bruni, C. Germani and R. Maartens, "Gravitational collapse on the brane," Phys. Rev. Lett. 87231302 (2001) [arXiv:gr-qc/0108013].

[35] R. Gregory, "Black string instabilities in anti-de Sitter space," Class. Quant. Grav. 17, L125 (2000) [arXiv:hep-th/0004101].

[36] G. Horowitz and K. Maeda, "Fate of the black string instability," Phys. Rev. Lett. 87131301 (2001) [arXiv:hep-th/0105111].

[37] D. Marolf, "On the fate of black string instabilities: An observation," Phys. Rev. D71 127504 (2005) [arXiv:hep-th/0504045]. 
[38] D. Garfinkle, L. Lehner and F. Pretorius, "A numerical examination of an evolving black string horizon," Phys. Rev. D71 064009 (2005) [arXiv:gr-qc/0412014].

[39] B. Kol, "The phase transition between caged black holes and black strings: A review," Phys. Rept. 422, 119 (2006) [arXiv:hep-th/0411240].

[40] R. Emparan, G. T. Horowitz and R. C. Myers, "Exact description of black holes on branes," JHEP 0001, 007 (2000) [arXiv:hep-th/9911043].

[41] S. Gubser and I. Mitra, "Instability of charged black holes in anti-de Sitter space," [arXiv:hep-th/0009126]

[42] R. Emparan, G. T. Horowitz and R. C. Myers, "Black holes radiate mainly on the brane," Phys. Rev. Lett. 85, 499 (2000) [arXiv:hep-th/0003118]. 